\title{
MCI Cancellation for Multicode Wideband CDMA Systems
}

\author{
Jun Chen, Jiangzhou Wang, Senior Member, IEEE, and Mamoru Sawahashi, Member, IEEE
}

\begin{abstract}
Multicode code division multiple access (CDMA) is a new transmission scheme for flexible and high-speed data communications. The basic idea of multicode CDMA is to assign multiple channelization codes to any given user. If these channel codes are orthogonal, the self-interference among them can be eliminated in an additive white Gaussian noise (AWGN) channel. However, in a multipath environment, these intrauser signals from different delay paths no longer maintain orthogonality and, thus, cause interference, i.e., multicode interference (MCI), to each other. In high-speed data networks, where the number of users is much less than in the voice networks, the MCI may represent a large portion of the total interference and has great impact on the system performance. In this paper, a complex spreading multicode wideband CDMA receiver with RAKE structure and multistage MCI cancellation is studied. By pilot aided channel estimation, the MCI associated with the reference user is regenerated and subtracted from the received signal by a cancellation factor of $\lambda$. A complete and consolidated theoretical analysis is presented to show that the system performance is significantly improved by the MCI cancellation. The optimal cancellation factor in the $k$ th stage is approximated by $\lambda_{\text {opt }}^{(k)} \leq 1-2 P_{e}^{(k-1)}$, where $P_{e}^{(k-1)}$ is the error probability of the $(k-1)$ th stage. The optimal value of each stage can be chosen from 0.5 to 0.85 for a wide range of signal to noise ratios.
\end{abstract}

Index Terms-Channel estimation, complex spreading, interference cancellation, multicode code division multiple access, RAKE receiver.

\section{INTRODUCTION}

$\mathbf{M}$ ULTICODE code division multiple access (CDMA) has been introduced as a new transmission scheme for high speed and flexible data rate communications over wireless channels [1]-[3]. The basic idea of multicode CDMA is to split the user data into a number of streams and use parallel orthogonal channel codes to modulate. As described in the $3 \mathrm{G}$ standards of wideband CDMA (W-CDMA) uplink physical layer, together with the orthogonal variable spreading factor (OVSF) method, multicode CDMA can provide data service up to the rate of 2 Mbps [3]. The performance of multicode CDMA system has been addressed in several papers [4]-[8].

The introduction of multicode causes several problems. One is the high envelope variation, which results from a linear sum of signals over multicode channels. There have been a number of methods proposed to combat such envelope fluctuation, e.g.,

Manuscript received December 15, 2000; revised June 6, 2001. This work was supported by NTT DoCoMo, Japan.

J. Chen and J. Wang are with Department of Electrical and Electronic Engineering, University of Hong Kong, Hong Kong (e-mail: junchen@eee.hku.hk; jwang@eee.hku.hk).

M. Sawahashi is with Wireless Research Laboratories, NTT DoCoMo, Inc., Kanagawa 239-8536, Japan (e-mail: sawahasi@ mlab.yrp.nttdocomo.co.jp).

Publisher Item Identifier S 0733-8716(02)01002-8. precoding [9] and predistortion [10], [11]. However, a convenient method is to use complex spreading, which can effectively suppress the envelope variation without introducing extra circuits or loss in information rate [12]. In this paper, complex spreading is adopted with the development of a unique representation of the complex signal. Another problem of multicode is the self-interference caused by the different delays of the intrauser signal in a multipath environment. These intrauser multicode signals have certain characteristics: synchronous, suffering the same fading for a given path and the channel codes associated with the reference user are known in both downlink and uplink. These features may be exploited to improve the receiver performance. That is, certain multicode detection techniques may be employed to achieve better performance.

Interference cancellation (IC) is to attempt the removal of the multiuser interference from each user's received signal before making final data decisions. IC has been vastly investigated [13]-[16]. The application of IC in multicode CDMA has also been suggested in some publications [17]-[21]. However, the following issues are not sufficiently addressed. First, in most publications, the system performance is examined by simulations rather than analysis, thus, more insightful study is necessary. Second, the signal with very large bandwidth in the next generation communication system shall confront with a large number of multipaths. Most publications assume that the number of RAKE receiver fingers is equal to the number of resolvable paths, and full cancellation is achieved. However, in any practical system, the number of RAKE fingers is limited and cannot be chosen arbitrarily. That is, the multipath interference cannot be completely cancelled. Third, IC requires the knowledge of channel parameters, e.g., amplitudes and phases. However, the channel estimation using pilot symbols or data symbols suffers a lot from the multipath and multiaccess interference and cannot be accurately obtained, which may greatly degrade the system performance. Fourth, since the tentative data decision for MCI regeneration may be not completely reliable, thus, it will be useful to use partial cancellation to mitigate the negative effects of incorrect decisions. [14], [15] studied the effect of partial cancellation by experiments and simulations. In this paper, the optimal partial MCI cancellation is analytically investigated and a multistage receiver with RAKE structure is presented. A consolidated theoretical analysis is given for system performance evaluation.

Basically, multiuser IC is used only in the uplink of the CDMA system, because in downlink, the mobile stations do not know the spreading codes of other users. However, in the case of multicode systems, the channel codes of the reference user are known in both uplink and downlink, thus, it is possible 


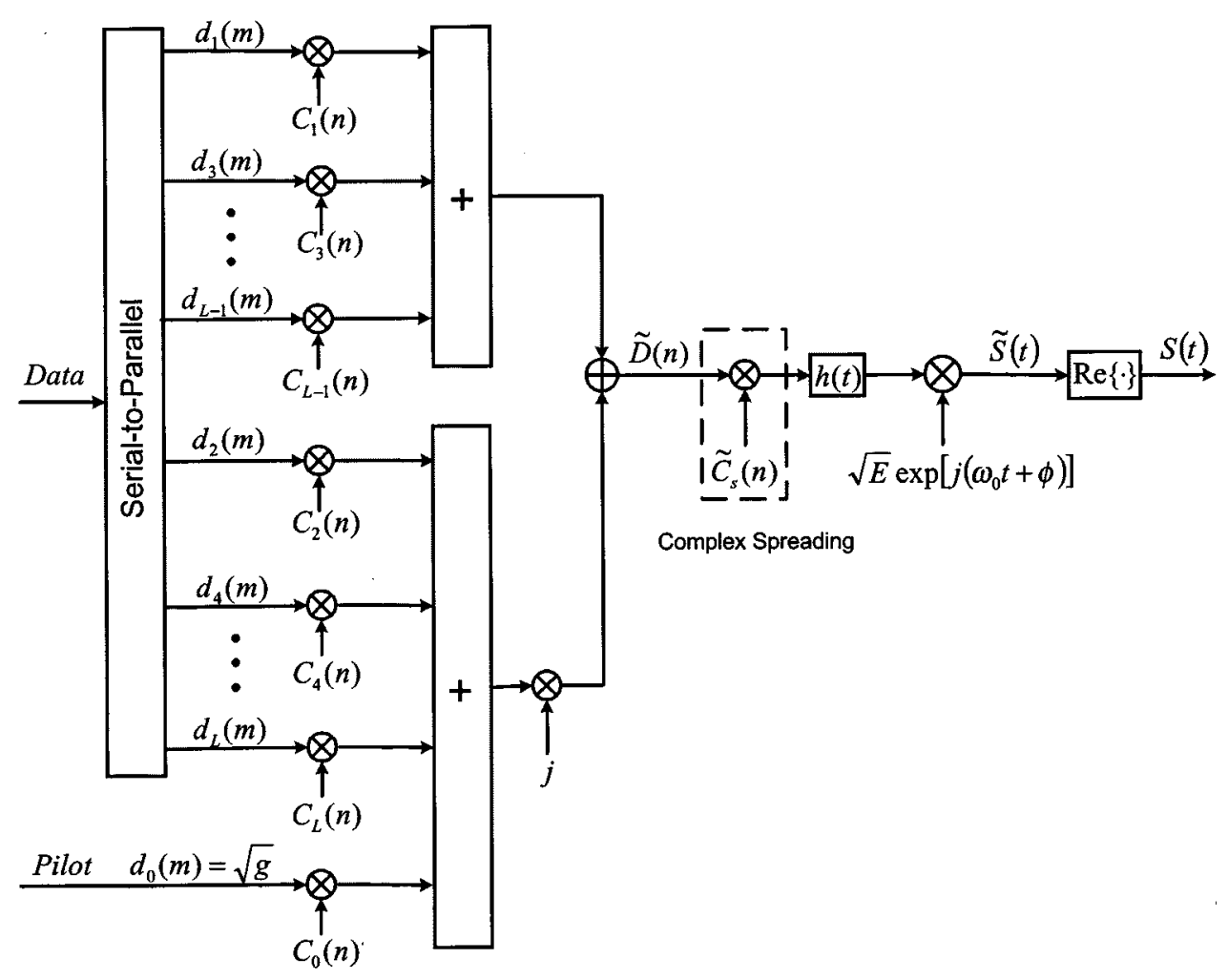

Fig. 1. Transmitter block diagram of the multicode CDMA system.

to utilize MCI cancellation in mobile stations as well. The uplink transmission model is used in this paper. However, the analysis and results can be applied to the downlink transmission as well.

Since multicode is only used for high-speed data transmission with high transmit power, the number of active users supported by the system is small. For instance, in 3GPP [3], when the number of data channels is six with a spreading factor (SF) of four in each code, only the multicode user is permitted for transmission in the whole system. That is, the number of active users in the system is one. Therefore, for simple analysis, only one user (i.e., the multicode user) is considered. However, even if there are other interfering users in the system, they can be suppressed by other methods, such as antenna arrays [22].

This paper is organized as follows. In Section II, the multicode CDMA system model is presented, which includes the transmitter diagram, a multipath channel model and detailed descriptions of the receiver structure. The analysis of the MCI cancellation receiver is given in Section III, including the decision statistics with and without MCI cancellation. The system performance is given in Section IV by means of bit-error rate (BER) and numerical results are discussed in Section V. Finally, conclusions are drawn in Section VI.

\section{Multicode CDMA System Models}

\section{A. Transmitter Diagram}

Consider the uplink of a single-cell multicode CDMA system. In a general case, any user in the system may use multicode to transmit high-speed data or may use a single code to transmit voice and low-speed data. Here, only one multicode user is assumed. Fig. 1 shows the transmitter block diagram of the multicode user, where $L+1$ denotes the number of code-channels. Note that one code (i.e., zeroeth code) with a large SF is for pilot channel, whereas other $L$ codes with small SF are for data channels. The ratio, $g$, is defined as the power ratio of pilot channel to one data channel. The user data is first split into $L$ parallel streams, i.e., serial to parallel conversion, then all streams are multiplied by corresponding orthogonal codes. This is so called the spreading or channelization process and the data symbols are transformed into chip streams. The streams are divided into two groups. In one group, the data streams, together with the pilot channel, are added to form the $Q$-branch data (imaginary part of the spreading data), whereas data streams in another group are added to form the $I$-branch data (real part of the spreading data). It is worth noting that any pair of data channels on the $I$ and $Q$ branches may use the same channel code, as specified in the standard of W-CDMA uplink physical layer of 3GPP [3], and the $I$ - and $Q$-data are distinguished by the following complex scrambling code. However, in this paper, all the $I$ - and $Q$-channels use different codes. But the system performance and models are identical for both cases.

The $I$ and $Q$ branches are treated as complex spreading data $\tilde{D}(n)$ and multiplied by a complex scrambling sequence $\widetilde{C}_{s}(n)$, which consists of two binary sequences, i.e., $\tilde{C}_{s}(n)= \pm 1 \pm j$. Note that hereinafter, the symbol " $\sim$ " is used to denote a complex variable. After scrambling, the resultant complex signal is passed through the pulse-shaping filter with impulse response, $h(t)$, and then multiplied with a complex carrier $\sqrt{E}\left[j\left(\omega_{0} t+\right.\right.$ 
$\phi)]$, where $E$ is chip energy, $\omega_{0}$ is angle frequency and $\phi$ is carrier phase. The real part of the modulated signal is transmitted and given by

$$
\begin{aligned}
& S(t)= \operatorname{Re}\{\tilde{S}(t)\} \\
&=\operatorname{Re}\left\{\sqrt{E} \sum_{n=-\infty}^{\infty} \tilde{D}(n) \cdot \tilde{C}_{s}(n)\right. \\
&\left.\cdot h\left(t-n T_{c}\right) \cdot \exp \left[j\left(\omega_{0} t+\phi\right)\right]\right\}
\end{aligned}
$$

where $\operatorname{Re}\{\cdot\}$ stands for real part and $\tilde{D}(n)$ is the complex spreading data, of the form

$$
\tilde{D}(n)=\sum_{l=1,3, \tilde{5}, \ldots} d_{l}(m) C_{l}(n)+j \sum_{l=0,2,4, \ldots} d_{l}(m) C_{l}(n),
$$

where $d_{l}(m)$ is the data sequence in $l$ th subchannel with $d_{0}(m)=\sqrt{g}$ and $d_{l}(m)= \pm 1$ for $l \neq 0$, and $m=\lfloor n / N\rfloor$, where $\lfloor\cdot\rfloor$ stands for the integer part and $N$ is the SF, $C_{l}(n)$ is the orthogonal channel code for $l$ th channel. Note that the pilot channel has a large SF $N=N_{0}$, while data channels take a small $\mathrm{SF} N=N_{1}$.

\section{B. Channel Model}

One of the basic features of the next-generation mobile communication system is the occupation of large bandwidth. When such a signal with large bandwidth is transmitted over wireless channels, it is very likely to demonstrate the characteristic of a large number of multipaths, especially in urban environment. A general multipath channel model is the tapped delay line model, with the complex low-pass equivalent impulse response that can be written as

$$
h_{\mathrm{ch}}(t)=\sum_{p=0}^{P-1} \alpha_{p}(t) \delta\left[t-\tau_{p}(t)\right] e^{j \theta_{p}(t)}
$$

where $P(P \geq 1)$ is the number of resolvable propagation paths. $\alpha_{p}(t) e^{j \theta_{p}(t)}$ and $\tau_{p}(t)$ are the complex fading factor and propagation delay, respectively, of the $p$ th path. It is assumed that all paths are independent. That is, all random variables in (3) are independent for $p$. An exponential multipath intensity profile (MIP) is assumed, i.e., $\Omega_{p}=E\left\{\alpha_{p}^{2}\right\}=e^{-p \delta}$, where $\delta$ is the rate of exponential decay of the path energy.

Therefore, the received signal is of the form

$$
R(t)=\eta(t)+\operatorname{Re}\left\{\sum_{p=0}^{P-1} \alpha_{p}(t) \tilde{S}\left[t-\tau_{p}(t)\right] e^{j \theta_{p}(t)}\right\}
$$

where $\eta(t)$ is the background additive white Gaussian noise (AWGN) with double-side power spectrum density $\eta_{0} / 2$.

Note that $\alpha_{p}(t)$ can be Rayleigh, Rician, or Nakagami distributed, depending on a specific channel model. $\alpha_{p}(t)$ is assumed to be Nakagami in this paper, because the Nakagami distribution is more versatile and more adequate to describe different fading situations. Accordingly, the probability density function (pdf) of $\left\{\alpha_{p}\right\}$ is given by

$$
p\left(\alpha_{p}\right)=2\left(\frac{m_{p}}{\Omega_{p}}\right)^{m_{p}} \frac{\alpha_{p}^{2 m_{p}-1}}{\Gamma\left(m_{p}\right)} \exp \left(-\frac{m_{p}}{\Omega_{p}} \alpha_{p}^{2}\right), \quad \alpha_{p} \geq 0
$$

where $m_{p}=\left(\Omega_{p}^{2} /\left(E\left\{\left(\alpha_{p}^{2}-\Omega_{p}\right)^{2}\right\}\right)\right)$ and $\Gamma(x)=$ $\int_{0}^{\infty} t^{x-1} e^{-t} d t$ is the Gamma function. Furthermore, if the Nakagami distribution in each path is such that the ratio $\left(m_{p} / \Omega_{p}\right)$ is the same for all paths, it can be shown that $\zeta=\sum_{p=0}^{P-1} \alpha_{p}^{2}$ has the gamma pdf [23]

$$
p(\zeta)=\left(\frac{m_{T}}{\Omega_{T}}\right)^{m_{T}} \frac{\zeta^{m_{T}-1}}{\Gamma\left(m_{T}\right)} \exp \left(-\frac{m_{T}}{\Omega_{T}} \zeta\right), \quad \zeta \geq 0
$$

where $\Omega_{T}=\sum_{p=0}^{P-1} \Omega_{p}=\sum_{p=0}^{P-1} E\left\{\alpha_{p}^{2}\right\}$ and $m_{T}=$ $\sum_{p=0}^{P-1} m_{p}$.

\section{Receiver Structure}

The receiver actually consists of several stages with similar structure. The overall structure is shown in Fig. 2, and the detailed block diagram of each stage is shown in Fig. 3. The basic idea is to make tentative decisions of the data symbols in the first stage using the conventional RAKE receiver. The tentative decisions are used to reconstruct MCI that to be canceled in the second stage, and secondary decisions are used in third stage to further cancel the interference, and so forth. Note that the front-end circuit (before sampling) is shared for all stages. As in any digital processing systems, the received signal is first downconverted to baseband, then passed through a pulse-matching filter, whose impulse response is the conjugate of the impulse response of the transmitter filter. The output is sampled at the chip rate $1 / T_{c}$, and these signal samples are fed to every stage correspondingly.

In all stages, a user-dedicated pilot channel is used to facilitate coherent reception. Descrambled samples are multiplied by a known pilot channel code, $-j C_{0}\left(n-n_{r}\right)$, and summed over $N_{0}$ chips to obtain an unbiased channel estimate. This estimate is noisy due to the multipath interference and a low-pass filter (LPF) is utilized to reduce the noise. In order to track the fading variation effectively, the cutoff frequency of the filter should be much larger than the maximum Doppler frequency $f_{d}$ of the wireless channel. In this study, a $N_{p}$-tap finite impulse response (FIR) filter is adopted to suppress the noise with assumption $\left(1 / N_{0} N_{P} T_{c}\right) \gg f_{d}$

In the first stage (without MCI cancellation), the sampled signal, $\tilde{u}_{r}(n)$, is first descrambled by multiplying conjugate of the scrambling code $\tilde{C}_{s}^{*}\left(n-n_{r}\right)$, then fed into a bank of RAKE processing units. There are $P$ multipath components in the received signal, however, only the strongest $R$ paths are processed, i.e., the number of RAKE fingers is $R(R \leq P)$. In each RAKE finger, the descrambled samples are multiplied by individual channel code $(-j)^{\overline{l+1}} C_{l}\left(n-n_{r}\right)$, where $\overline{l+1}=(l+1) \bmod 2$, then integrated and dumped (I\&D). After that, the outputs of all data channels are multiplied by the conjugate of corresponding channel estimate from pilot symbols to complete coherent demodulation and maximum ratio combining. Each finger produces $L$ outputs and all outputs of $R$ fingers are added correspondingly to form $L$ decision statistics for the $L$ parallel data symbols.

In the second stage $\left(\lambda^{(2)} \neq 0\right)$, the MCI is first reconstructed for each RAKE finger, using previous data decisions and 


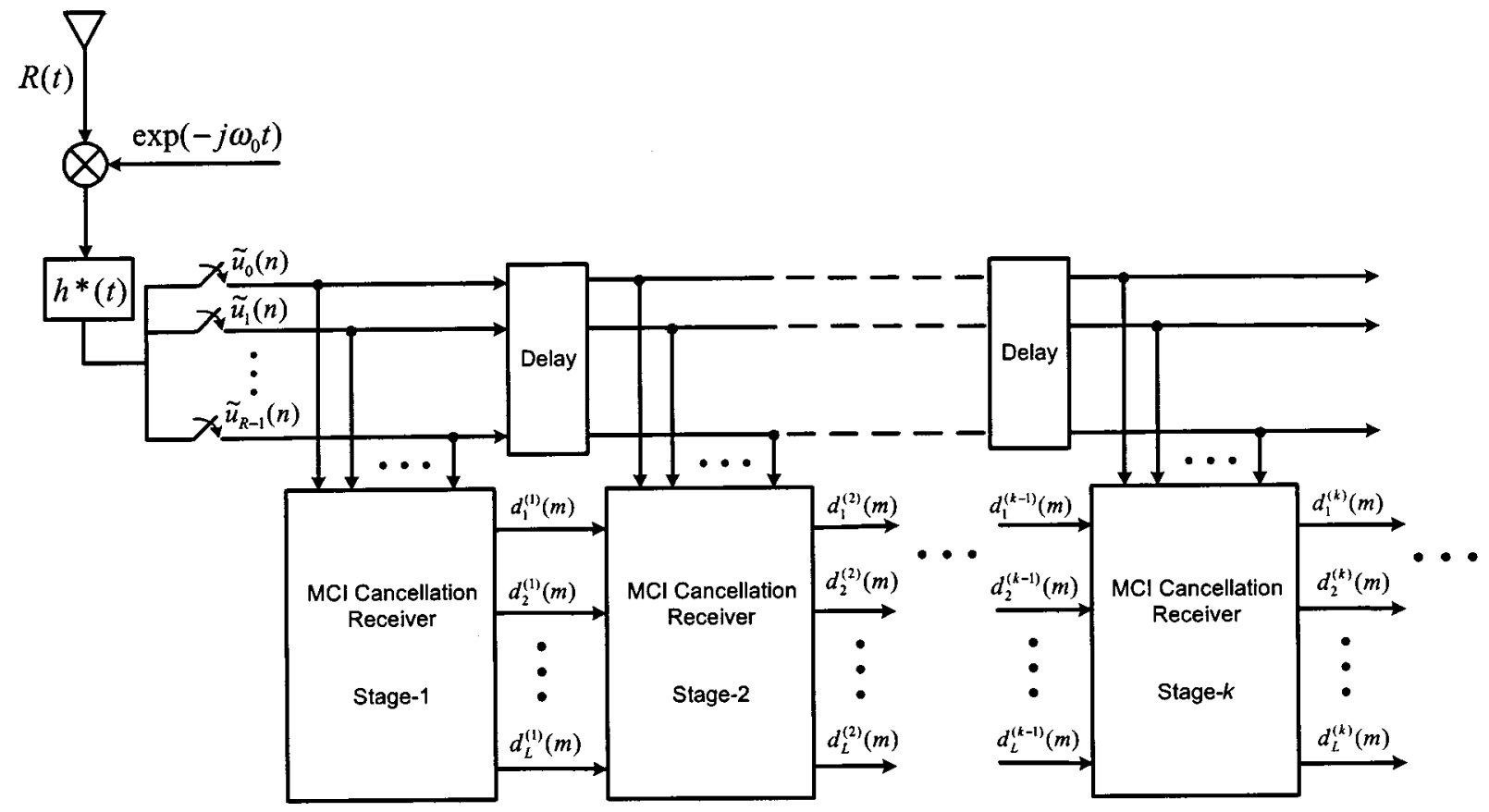

Fig. 2. The overall structure of the multistage $\mathrm{MCI}$ cancellation receiver.

channel estimates. For each data channel, the total number of interference terms should be $(L+1) P-1$. However, since those multicode signals that arrive at the same path are orthogonal and do not cause interference, only $(L+1)(P-1)$ terms of interference need to be eliminated. Due to implementation limits of RAKE receiver, only the parameters of $R$ strongest paths are estimated, so that only $(L+1)(R-1)$ terms of interference components could be regenerated and cancelled. The detailed block diagram of the interference regenerator in $r$ th finger is shown in Fig. 4, where $\left\{d_{l}^{(k-1)}(m)=d_{l}^{(1)}(m), l=1, \ldots, L\right\}$ are the tentative decisions from the first stage. These tentative data decisions $\left\{d_{l}^{(1)}(m)\right\}$ and scaled channel estimates $\left\{\widetilde{\gamma}_{p}^{(1)}(m) /\left(2 \sqrt{g} N_{o}\right)\right\}$ are multiplied by channel codes and scrambling sequences with different delay. Finally, a weighting factor $\lambda^{(k)}$, shown in Fig. 3, is introduced to control the weight of interference being removed. Because when the interference estimate is poor, e.g., the signal-to-noise ratio (SNR) is low, it is preferable not to cancel the entire amount of estimated interference but to remove only a part of the interference. After subtraction, the resultant signal is processed as in the first stage and secondary decisions are made.

Notice that in multipath environment, the path delay spread may be larger than one data symbol duration. Since the MCI is regenerated on chip level, the tentative data in the $\mathrm{MCI}$ regeneration units may be the current one, or the previous one, or even a few bits ahead. This operation is enabled by the fact that the multipath delay can be measured and tracked in system. An example of the time relation between the data bits in a multipath channel is shown in Fig. 5.

In order to obtain acceptable performance, more stages with MCI cancellation are necessary. The operations of the $k$ th stages are like that of the second stage.

\section{ANALYSIS OF MCI CANCELLATION}

\section{A. Decision Statistic in First Stage (Without MCI Cancellation)}

Assuming that acquisition and synchronization have been accomplished for the reference path, $r$, the path delay $\tau_{r}(t)$ is known, so that it is able to pick up the strongest $R$ paths. Therefore, the local spreading codes in the $r$ th finger are aligned with the $r$ th path of the received signal. In Fig. 3, the sampled output of the pulse-matching filter for the $r$ th finger is given by

$$
\begin{aligned}
\tilde{u}_{r}(n)= & \int_{n T_{c}+\tau_{r}^{\prime}(n)}^{(n+1) T_{c}+\tau_{r}^{\prime}(n)} R(t) \exp \left(-j \omega_{0} t\right) \\
& \times h^{*}\left[n T_{c}+\tau_{r}^{\prime}(n)-t\right] d t \\
= & \tilde{\eta}(n)+\sum_{p=0}^{P-1} \frac{1}{2} \sqrt{E} \alpha_{p}(n) R_{w}\left[\bar{\tau}_{p}(n)\right] \exp \left[j \varphi_{p}(n)\right] \\
& \cdot \tilde{D}\left(n-n_{p}\right) \cdot \tilde{C}_{s}\left(n-n_{p}\right) \\
= & \tilde{\eta}(n)+\sum_{p=0}^{P-1} \frac{1}{2} \sqrt{E} \tilde{\beta}_{p}(n) \cdot \tilde{D}\left(n-n_{p}\right) \cdot \tilde{C}_{s}\left(n-n_{p}\right)
\end{aligned}
$$

where for the sake of simple notation, $n T_{c}$ is replaced by $n$ hereafter, and $\tau_{r}^{\prime}(n)=\tau_{r}(n) \bmod T_{c}, n_{p}=$ $\left\lfloor\left[\tau_{p}(n)-\tau_{r}^{\prime}(n)\right] / T_{c}\right\rfloor, \tilde{\eta}(n)$ is the sampled noise component, and $\tilde{\beta}_{p}(n)$ is of the form

$$
\tilde{\beta}_{p}(n)=\alpha_{p}(n) R_{w}\left[\bar{\tau}_{p}(n)\right] \exp \left[j \varphi_{p}(n)\right]
$$

where $\varphi_{p}(n)=\phi+\theta_{p}(n)-\omega_{0} \tau_{p}(n)$ is the overall carrier phase offset. $\bar{\tau}_{p}(n)$ is the chip timing error given by $\bar{\tau}_{p}(n)=$ $\tau_{p}(n)-\tau_{r}^{\prime}(n)-n_{p} T_{c} . R_{w}(\bar{\tau})$ represents the effect of timing 


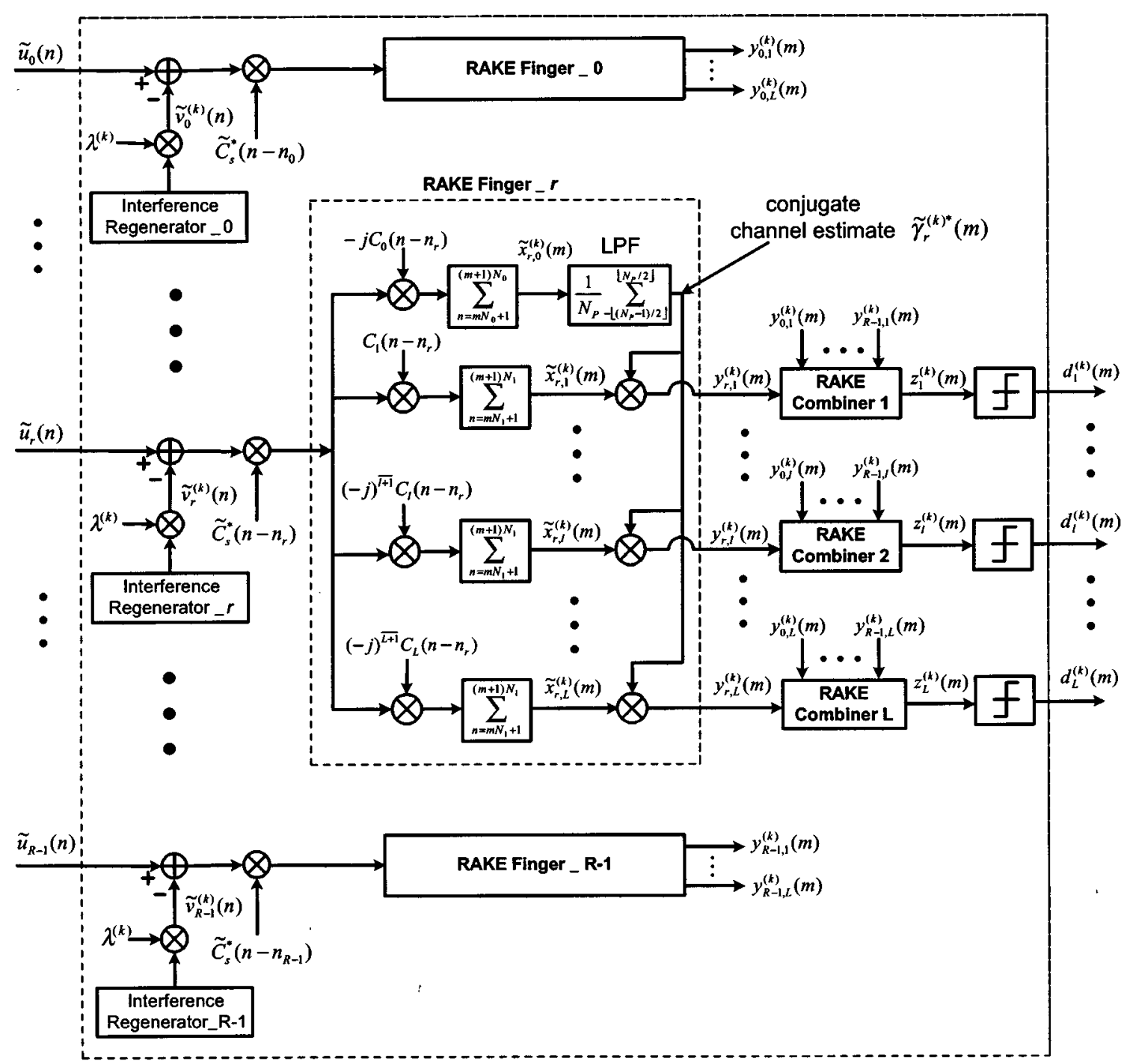

Fig. 3. The detailed block diagram of the $k$ th stage.

error $\bar{\tau}$ on the sampled output. If the impulse response of the shaping filter is limited to one $T_{c}$ interval, then we have [12]

$$
R_{w}(\bar{\tau})=\int_{-\infty}^{\infty}|H(f)|^{2} \cos (2 \pi f \bar{\tau}) d f
$$

where $H(f)$ is the frequency response of the pulse shaping filter.

It is assumed that for different path $p$, the fading amplitudes $\alpha_{p}(n)$ are independent, chip timing errors $\bar{\tau}_{p}(n)$ are independent identically distributed (i.i.d.) random variables uniformly distributed over $\left(0, T_{c}\right]$ except for $\bar{\tau}_{r}(n)=0$, carrier phase offsets $\varphi_{p}(n)$ are i.i.d random variables uniform over $(0,2 \pi]$. Accordingly, $\tilde{\beta}_{p}(n)$ in (8) can be modeled as a zero mean variable with variance

$$
\begin{aligned}
\operatorname{var}\left\{\tilde{\beta}_{p}(n)\right\} & =E\left\{\tilde{\beta}_{p}^{2}(n)\right\} \\
& =E\left\{\alpha_{p}^{2}(n)\right\} \cdot E\left\{R_{w}^{2}\left[\bar{\tau}_{p}(n)\right]\right\} \cdot E\left\{\exp ^{2}\left[j \varphi_{p}(n)\right]\right\} \\
& =\left(\frac{1}{2}+j \frac{1}{2}\right) \Delta \Omega_{p}
\end{aligned}
$$

where $\Omega_{p}=E\left\{\alpha_{p}^{2}(n)\right\}$ is the channel parameter given in the channel model, $\Delta=E\left\{R_{w}^{2}\left[\tau_{p}(n)\right]\right\}$ is related to the frequency transform function of pulse-shaping filter, and $\Delta=(2 / 3)$ for rectangle pulse shaping filter, $E\left\{\exp ^{2}\left[j \varphi_{p}(n)\right]\right\}$ is defined as $E\left\{\cos ^{2} \varphi_{p}(n)\right\}+j E\left\{\sin ^{2} \varphi_{p}(n)\right\}=(1 / 2)+j(1 / 2)$. Notice that the complex variables used in this paper are for the purpose of avoiding ponderous notations. In most cases, the real and imaginary parts are corresponding to the $I$ and $Q$ components in processing. Hence, in mathematical operations, the real and imaginary parts will be dealt individually.

The output of the I\&D correlator in the data branch $l(l \geq 1)$ of the finger $r$ at the first stage is

$\tilde{x}_{r, l}^{(1)}(m)=\sum_{n=m N_{1}+1}^{(m+1) N_{1}} \tilde{u}_{r}(n) \cdot \tilde{C}_{s}^{*}\left(n-n_{r}\right) \cdot(-j)^{\overline{l+1}} C_{l}\left(n-n_{r}\right)$

where $(-j)^{\overline{l+1}}=1$ and $-j$ for odd and even $l$, respectively. Assuming that the channel parameters remain constant over one symbol duration, i.e., $\tilde{\beta}_{p}(n)=\tilde{\beta}_{p}\left(m N_{1}\right)$ for $m N_{1}<n \leq(m+1) N_{1}$, it can be shown that the output, $\tilde{x}_{r, l}^{(1)}(m)$, consists of three distinct components: the desired 


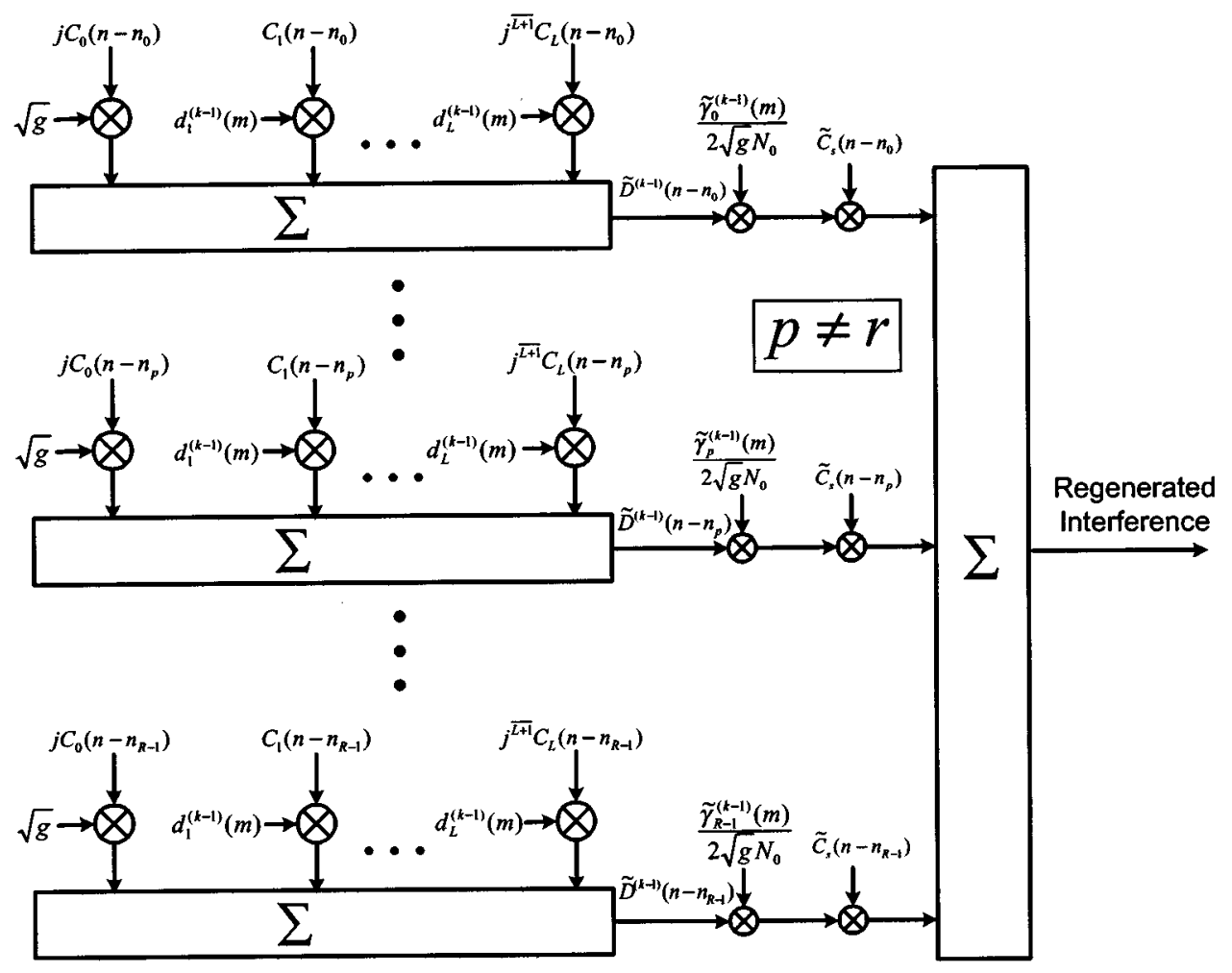

Fig. 4. Interference regeneration of the $r$ th finger in $k$ th stage.

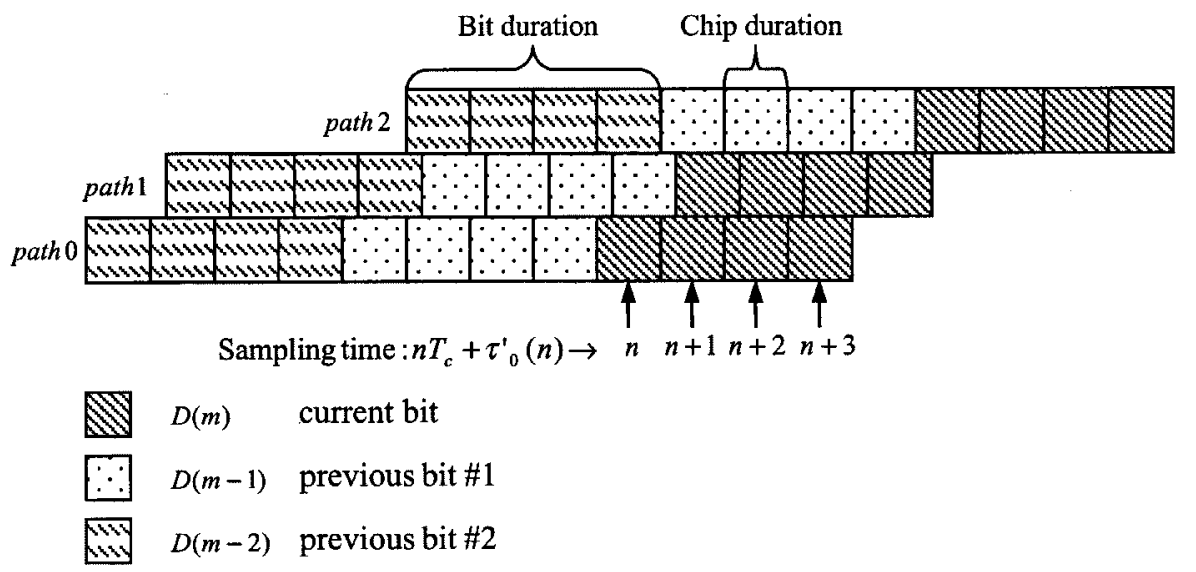

Fig. 5. Time relation of the data bits in the multipath channel.

signal component $\tilde{E}_{r, l}^{(1)}(m)$; the intrauser multicode interference $\tilde{I}_{r, l}^{(1)}(m)$; and the background AWGN component $\tilde{\eta}_{r, l}^{(1)}(m)$. That is

$$
\tilde{x}_{r, l}^{(1)}(m)=\tilde{E}_{r, l}^{(1)}(m)+\tilde{I}_{r, l}^{(1)}(m)+\tilde{\eta}_{r, l}^{(1)}(m) .
$$

Since the objective of this research is to reduce the variance of $\tilde{I}_{r, l}^{(1)}(m)$, it is separately listed as follows:

$$
\begin{aligned}
\tilde{I}_{r, l}^{(1)}(m)= & \sum_{\substack{p=0 \\
p \neq r}}^{P-1} \frac{1}{2} \sqrt{E} \tilde{\beta}_{p}\left(m N_{1}\right) \\
& \times \sum_{n=m N_{1}+1}^{(m+1) N_{1}} \tilde{D}\left(n-n_{p}\right) \cdot \tilde{C}_{s}\left(n-n_{p}\right) \\
& \cdot \tilde{C}_{s}^{*}\left(n-n_{r}\right) \cdot(-j)^{\overline{l+1}} C_{l}\left(n-n_{r}\right) .
\end{aligned}
$$

The variance of $\tilde{I}_{r, l}^{(1)}(m)$ is given by

$$
\operatorname{var}\left\{\tilde{I}_{r, l}^{(1)}(m)\right\}=(1+j) \Delta \frac{N_{1}}{2} \sum_{\substack{p=0 \\ p \neq r}}^{P-1} \Omega_{p}(L+g) E .
$$

In any finger of the RAKE receiver, the output of the top branch; namely, $\tilde{x}_{r, 0}^{(1)}(m)$, is the channel estimate from the pilot symbol. In order to reduce the error of channel estimate, $\tilde{x}_{r, 0}^{(1)}(m)$ is passed through a low-pass FIR filter with $N_{P}$ taps. For coherent detection and maximum ratio combining, the data estimate is multiplied by conjugate of the channel estimate. Assuming that the scrambling sequences are pure random sequences and channel parameters remain unchanged over $N_{P}$ symbols, one can have

$$
E\left\{y_{r, l}^{(1)}(m)\right\}=N_{0} N_{1} \sqrt{g} E \alpha_{r}^{2}\left(m N_{1}\right) d_{l}(m)
$$




$$
\begin{aligned}
\operatorname{var}\left\{y_{r, l}^{(1)}(m)\right\}= & \left(g N_{0}+\frac{N_{1}}{N_{P}}\right) \\
& \times N_{0} N_{1} E \alpha_{r}^{2}\left(m N_{1}\right) \Lambda^{(1)}+\frac{2 N_{0} N_{1}}{N_{P}}\left[\Lambda^{(1)}\right]^{2}
\end{aligned}
$$

where $\Lambda^{(1)}=\Delta(1 / 2) \sum_{p=0}^{P-1} \Omega_{p}(L+g) E+\eta_{0}$ is a statistic that gives the measurement of the overall interference in the first stage. The readers can refer to [12] for further computational details.

After RAKE combining, the decision statistic of data symbol in the $l$ th code channel is the sum of outputs in $R$ corresponding fingers

$$
z_{l}^{(1)}(m)=\sum_{r=0}^{R-1} y_{r, l}^{(1)}(m) .
$$

Because the variables in different paths are independent, the mean and variance of $z_{l}(m)$ are

$$
\begin{aligned}
E\left\{z_{l}^{(1)}(m)\right\}= & N_{1} N_{0} \sqrt{g} E \sum_{r=0}^{R-1} \alpha_{r}^{2}\left(m N_{1}\right) d_{l}(m) \\
= & N_{1} N_{0} \sqrt{g} E \zeta_{R} d_{l}(m) \\
\operatorname{var}\left\{z_{l}^{(1)}(m)\right\}= & \left(g N_{0}+\frac{N_{1}}{N_{P}}\right) N_{0} N_{1} E \Lambda^{(1)} \\
& \times \sum_{r=0}^{R-1} \alpha_{r}^{2}\left(m N_{1}\right)+\frac{2 N_{0} N_{1}}{N_{P}} \sum_{r=0}^{R-1}\left[\Lambda^{(1)}\right]^{2} \\
= & \left(g N_{0}+\frac{N_{1}}{N_{P}}\right) N_{0} N_{1} E \Lambda^{(1)} \zeta_{R} \\
& +\frac{2 R N_{0} N_{1}}{N_{P}}\left[\Lambda^{(1)}\right]^{2}
\end{aligned}
$$

where $\zeta_{R}=\sum_{r=0}^{R-1} \alpha_{r}^{2}\left(m N_{1}\right)$ is a random variable determined by the instantaneous amplitude fading.

In order to regenerate the multicode interference, a tentative decision of each data symbol is to be made in the first stage. For hard decision, the estimated data symbol in the first stage is

$$
d_{l}^{(1)}(m)=\operatorname{sgn}\left[z_{l}^{(1)}(m)\right] .
$$

Notice that the number of independent interference components in $y_{r, l}^{(1)}(m)$ is $P$, consequently, the number of interference terms in $z_{l}^{(1)}(m)$ is $P R$. When $P R$ is very large (which is true for general cases), $z_{l}^{(1)}(m)$ can be modeled as a Gaussian variable. Therefore an instantaneous error probability of this tentative decision is given by

$$
\begin{aligned}
P_{e}^{(1)} & =P_{e, l}^{(1)}\left(\zeta_{R}\right)=Q\left(\frac{\left|E\left\{z_{l}(m)\right\}\right|}{\sqrt{\operatorname{var}\left\{z_{l}(m)\right\}}}\right) \\
& =Q\left(\frac{\zeta_{R}}{\sqrt{\zeta_{R}\left(1+\frac{1}{g N^{\prime}}\right) \frac{\Lambda^{(1)}}{N_{1} E}+\frac{2 R}{g^{\prime}} \cdot\left(\frac{\Lambda^{(1)}}{N_{1} E}\right)^{2}}}\right)
\end{aligned}
$$

where $N^{\prime}=N_{P} \cdot N_{0} / N_{1},|x|$ denotes the absolute value and $Q(x)=\int_{x}^{\infty}(1 / \sqrt{2 \pi}) e^{-y^{2} / 2} d y$ is the $Q$-function. Note that the overall error probability is equal to that of each data channel.

\section{B. Decision Statistic in Second Stage (With MCI Cancellation)}

The regenerated interference of the $r$ th finger in the second stage is given by

$$
\begin{aligned}
& \tilde{v}_{r}^{(2)}(n)=\lambda^{(2)} \cdot \sum_{\substack{p=0 \\
p \neq r}}^{R-1} \frac{\tilde{\gamma}_{p}^{(1)}(m)}{2 \sqrt{g} N_{0}} \tilde{D}^{(1)}\left(n-n_{p}\right) \\
& \quad \tilde{C}_{s}\left(n-n_{p}\right), \quad 0 \leq r \leq R-1
\end{aligned}
$$

where $\quad \tilde{D}^{(1)}(n)=\sum_{l=1,3,5, \ldots} d_{l}^{(1)}(m) C_{l}(n)+$ $j \sum_{l=0,2,4, \ldots} d_{l}^{(1)}(m) C_{l}(n), 0 \leq l \leq L$, is the reconstructed complex spreading data using tentative decisions in the first stage. It is seen that the reconstructed interference is irrelevant to different subchannel $l$, and distinct only for different RAKE finger. This is because the multicode signals that arrive at the same delay are orthogonal to each other and the resultant interference is zero. Therefore, there are only $(L+1)(R-1)$ terms of interference components involved in cancellation.

The correlator output in the $l$ th data channel of the second stage is

$$
\begin{aligned}
\tilde{x}_{r, l}^{(2)}(m)= & \sum_{n=m N_{1}+1}^{(m+1) N_{1}}\left[\tilde{u}_{r}(n)-\tilde{v}_{r}^{(2)}(n)\right] \\
& \cdot \tilde{C}_{s}^{*}\left(n-n_{r}\right) \cdot(-j)^{\overline{l+1}} C_{l}\left(n-n_{r}\right) \\
= & \tilde{E}_{r, l}^{(2)}(m)+\tilde{I}_{r, l}^{(2)}(m)+\tilde{\eta}_{r, l}^{(2)}(m) \\
= & \tilde{E}_{r, l}^{(1)}(m)+\tilde{I}_{r, l}^{(2)}(m)+\tilde{\eta}_{r, l}^{(1)}(m)
\end{aligned}
$$

where the desired component $\tilde{E}_{r, l}^{(2)}(m)=\tilde{E}_{r, l}^{(1)}(m)$ and the noise component $\tilde{\eta}_{r, l}^{(2)}(m)=\tilde{\eta}_{r, l}^{(1)}(m)$, the only component different from that in the first stage is the MCI, i.e., $\tilde{I}_{r, l}^{(1)}(m) \rightarrow$ $\tilde{I}_{r, l}^{(2)}(m) . \tilde{I}_{r, l}^{(2)}(m)$ is of the form

$$
\begin{aligned}
\tilde{I}_{r, l}^{(2)}(m)= & \sum_{p=R}^{P-1} \frac{1}{2} \sqrt{E} \tilde{\beta}_{p}\left(m N_{1}\right) \sum_{n=m N_{1}+1}^{(m+1) N_{1}} \tilde{D}\left(n-n_{p}\right) \\
& \cdot \tilde{C}_{s}\left(n-n_{p}\right) \cdot \tilde{C}_{s}^{*}\left(n-n_{r}\right) \cdot(-j)^{\overline{l+1}} C_{l}\left(n-n_{r}\right) \\
& +\sum_{\substack{p=0 \\
p \neq r}}^{R-1} \sum_{n=m N_{1}+1}^{(m+1) N_{1}}\left[\frac{1}{2} \sqrt{E} \tilde{\beta}_{p}\left(m N_{1}\right) \tilde{D}\left(n-n_{p}\right)\right. \\
& \left.\quad-\lambda^{(2)} \cdot \frac{\tilde{\gamma}_{p}^{(1)}(m)}{2 \sqrt{g} N_{0}} \tilde{D}^{(1)}\left(n-n_{p}\right)\right] \\
& \cdot \tilde{C}_{s}\left(n-n_{p}\right) \cdot \tilde{C}_{s}^{*}\left(n-n_{r}\right) \cdot(-j)^{\overline{l+1}} C_{l}\left(n-n_{r}\right) \\
= & \tilde{I}_{r, l}^{(2)}(m)+\tilde{I}_{r, l}^{\prime \prime(2)}(m) .
\end{aligned}
$$

Notice that after cancellation, the MCI still consists of two residual parts. The first part is the interference that is beyond the $R$ strongest paths, which is not being cancelled at all. The variance of this part does not change with the increase of stages, and is given by

$$
\operatorname{var}\left\{\tilde{I}_{r, l}^{(2)}(m)\right\}=(1+j) \Delta \frac{N_{1}}{2} \sum_{p=R}^{P-1} \Omega_{p}(L+g) E .
$$

The second part is the residual interference within the $R$ paths, which is due to two factors. First is the error of scaled channel estimate, that is, $\left(\tilde{\gamma}_{p}^{(1)}(m) / 2 \sqrt{g} N_{0}\right) \neq$ 
$(\sqrt{E} / 2) \tilde{\beta}_{p}\left(m N_{1}\right), 0 \leq p \leq R-1$. The second factor is the tentative data decision errors from the first stage, that is, $d_{l}^{(1)}(m) \neq d_{l}(m), l=1,2, \ldots, L$. Notice that in MCI regeneration, the tentative data is multiplied by the channel estimate, so the overall error is essentially the product of data error and channel estimate error. It is seen from the previous description that the data error results from the ongoing $N_{1}$ chips, while the channel estimate is the average over $N_{P} \cdot N_{0}$ chips. Due to the fact of $N_{P} \cdot N_{0} \gg N_{1}$ in practical system, (for instance, $N_{P} \cdot N_{0}=512$ and $N_{1}=4$ ), these two errors have a weak correlation, although they are not totally uncorrelated. Therefore, the interference can be approximated as the summation of the interferences in two cases. First is the interference where the channel estimation is error free, while the tentative data decisions have errors with error rate $P_{e}^{(1)}$ given by (21). Second is the interference where the tentative data decisions are error free, while the scaled channel estimate has deviation (variance)

$$
\begin{aligned}
\operatorname{var}\left\{\frac{\tilde{\gamma}_{p}^{(1)}(m)}{2 \sqrt{g} N_{0}}\right\} & =(1+j) \frac{\Lambda^{(1)}}{4 g N_{P} N_{0}} \\
& =\left(\frac{1}{8}+j \frac{1}{8}\right) \Delta E\left(\sigma_{p}^{(1)}\right)^{2}
\end{aligned}
$$

where

$$
\left(\sigma_{p}^{(1)}\right)^{2}=\frac{L+g}{g N_{P} N_{0}} \sum_{p=0}^{P-1} \Omega_{p}+\frac{\eta_{0}}{g E N_{P} N_{0}} .
$$

Notice that $\left(\sigma_{p}^{(1)}\right)^{2}$ is approximately irrelevant to the subscript $p$, although it represents the variance of channel estimate of the pth path.

When $B^{(1)}$ out of $L$ data channel decisions are incorrect at the first stage, the MCI caused by the error decisions is increased by $\left(1+\lambda^{(2)}\right)^{2}$ times. For the remaining correct $\left(L-B^{(1)}\right)$ decisions, the interference is reduced by $\left(1-\lambda^{(2)}\right)^{2}$ times. Moreover, the channel estimation error is additive to this interference, no matter whether the data decision are correct or not. Note that there is no tentative decision error in the pilot channel. Therefore, the variance of this residual interference is given by

$$
\begin{aligned}
& \operatorname{var}\left\{\tilde{I}_{r, l}^{\prime \prime(2)}(m)\right\}=(1+j) \Delta \frac{N_{1} E}{2} \\
& \times\left(\sum_{\substack{p=0 \\
p \neq r}}^{R-1} \Omega_{p} \times\left[\left(1+\lambda^{(2)}\right)^{2} \bar{B}^{(1)}+\left(1-\lambda^{(2)}\right)^{2}\left(L-\bar{B}^{(1)}\right)\right]\right. \\
& \left.\quad+\sum_{\substack{p=0 \\
p \neq r}}^{R-1}\left(\lambda^{(2)}\right)^{2}\left(\sigma_{p}^{(1)}\right)^{2}(L+g)\right) \\
& =(1+j) \Delta \frac{N_{1} E}{2} \\
& \quad \times\left(\sum_{\substack{p=0 \\
p \neq r}}^{R-1} \Omega_{p}\left[4 \lambda^{(2)} \bar{B}^{(1)}+\left(1-\lambda^{(2)}\right)^{2} L\right]\right. \\
& \quad+\sum_{\substack{p=0 \\
p \neq r}}^{R-1}\left(\lambda^{(2)}\right)^{2}\left(\sigma_{p}^{(1)}\right)^{2}(L+g)
\end{aligned}
$$

where $\bar{B}^{(1)}=E\left\{B^{(1)}\right\}=L P_{e}^{(1)}$ is the average number of error bits at the first stage. Strictly speaking, $B^{(1)}$ should not be averaged here. Rather, subsequent conditional error probability should be averaged over $B^{(1)}$. However, since the tentative data decision errors are not independent from each other, it is very difficult to obtain the distribution of $B^{(1)}$. Moreover, as shown in Fig. 5, when MCI is regenerated, the data is not restricted to the data in current position, but also can be the previous one or a few bits ahead. When the path delay changes with the time, the relative position is changed. So, $B^{(1)}$ is not the error pattern of the $L$-bits data for one specific moment, but rather represents an average error probability of $L$-channels for a small range of time. Therefore, $\bar{B}^{(1)}$ is used instead of $B^{(1)}$. This approximation not only simplifies the analysis, but also makes sense in this situation.

The total variance of MCI after cancellation in the second stage is given by

$$
\operatorname{var}\left\{\tilde{I}_{r, l}^{(2)}(m)\right\}=\operatorname{var}\left\{\tilde{I}_{r, l}^{\prime(2)}(m)\right\}+\operatorname{var}\left\{\tilde{I}_{r, l}^{\prime \prime(2)}(m)\right\}
$$

Similar to $\widetilde{x}_{r, l}^{(1)}(m)$, the variance of $\tilde{x}_{r, l}^{(2)}(m)$ can be written as

$$
\begin{aligned}
\operatorname{var}\left\{\tilde{x}_{r, l}^{(2)}(m)\right\} & =\operatorname{var}\left\{\tilde{I}_{r, l}^{(2)}(m)\right\}+\operatorname{var}\left\{\tilde{\eta}_{r, l}^{(2)}(m)\right\} \\
& =(1+j) N_{1} \Lambda^{(2)}
\end{aligned}
$$

where $\Lambda^{(2)}$ is given by

$$
\begin{aligned}
\Lambda^{(2)}=\frac{\Delta E}{2}( & \sum_{p=R}^{P-1} \Omega_{p}(L+g) \\
& +\sum_{\substack{p=0 \\
p \neq r}}^{R-1} \Omega_{p}\left[4 \lambda^{(2)} \bar{B}^{(1)}+\left(1-\lambda^{(2)}\right)^{2} L\right] \\
& \left.+\sum_{\substack{p=0 \\
p \neq r}}^{R-1}\left(\lambda^{(2)}\right)^{2}\left(\sigma_{p}^{(1)}\right)^{2}(L+g)\right)+\eta_{0} \\
\approx \frac{\Delta E}{2}\left(\sum_{p=R}^{P-1} \Omega_{p}(L+g)\right. & +\sum_{p=0}^{R-1} \Omega_{p}\left[4 \lambda^{(2)} \bar{B}^{(1)}+\left(1-\lambda^{(2)}\right)^{2} L\right] \\
& \left.+\sum_{p=0}^{R-1}\left(\lambda^{(2)}\right)^{2}\left(\sigma_{p}^{(1)}\right)^{2}(L+g)\right)+\eta_{0}
\end{aligned}
$$

$\Lambda^{(2)}$ is the measurement of total interference after MCI cancellation in the second stage. Similar to the first stage, the mean and variance of the decision statistic in the second stage can also be written in the forms of (18) and (19), but with $\Lambda^{(1)} \rightarrow \Lambda^{(2)}$. Therefore, it is possible to calculate the error performance of the second stage on the basis of the first stage. 


\section{Optimal Partial MCI Cancellation}

In view of the fact that the tentative decisions are not completely reliable, a possible way to improve the performance is not to attempt to cancel interference completely, but partially. This can be accomplished by the weighting factor $\lambda^{(2)}$, shown in Fig. 3. The second part of the residual interference after cancellation is rewritten as

$$
\begin{aligned}
\operatorname{var}\left\{\tilde{I}_{r, l}^{\prime \prime(2)}(m)\right\}= & (1+j) \Delta \frac{N_{1} E}{2} \\
& \times\left(\sum_{\substack{p=0 \\
p \neq r}}^{R-1} \Omega_{p}\left[4 \lambda^{(2)} \bar{B}^{(1)}+\left(1-\lambda^{(2)}\right)^{2} L\right]\right. \\
& \left.+\sum_{\substack{p=0 \\
p \neq r}}^{R-1}\left(\lambda^{(2)}\right)^{2}\left(\sigma_{p}^{(1)}\right)^{2}(L+g)\right) .
\end{aligned}
$$

It can be seen from the first term of the right hand side of (32) that in the presence of the weighting factor $\left(\lambda^{(2)} \neq 0\right)$, interference cannot be completely removed even though tentative data decisions are correct. When $\lambda^{(2)}$ is small, interference caused by both incorrect tentative data decisions and imperfect channel estimation is small, whereas the interference introduced by correct tentative decision is large. Therefore, an optimal value of $\lambda^{(2)}$ should exist according to different channel conditions. That is, when channel condition is good (the error rate of tentative decision is low), a large value of $\lambda^{(2)}$ is desirable (close to one). However, when the channel condition is poor (the noise and interference level is high), a smaller value of $\lambda^{(2)}$ is preferable.

The optimal $\lambda^{(2)}$ is to minimize the variance given by (32). Thus, by letting $\left(d \operatorname{var}\left\{\tilde{I}_{r, l}^{\prime \prime(2)}(m)\right\} / d \lambda^{(2)}\right)=0$, it is readily to have

$$
\lambda_{\mathrm{opt}}^{(2)}=\frac{\sum_{\substack{p=0 \\ p \neq r}}^{R-1} \Omega_{p}\left(L-2 \bar{B}^{(1)}\right)}{\sum_{\substack{p=0 \\ p \neq r}}^{R-1} \Omega_{p} L+\sum_{\substack{p=0 \\ p \neq r}}^{R-1}\left(\sigma_{p}^{(1)}\right)^{2}(L+g)} .
$$

This result agrees with the above discussion on how to choose the optimal value of $\lambda^{(2)}$, which should be in the range $\lambda^{(2)}<1$. It is seen from (33) that when $2 \bar{B}>L, \lambda_{\text {opt }}^{(2)}<0$. This can be instinctively explained as following. The regenerated interference component from an error decision is a negative term compared with that from a correct data decision. If more than half of the data decisions in the first stage are incorrect $(\bar{B}>L / 2)$, then the overall regenerated interference would be a negative one. In this case, to subtract the regenerated interference cannot reduce the interference at all, but increase the interference. Thus, the optimal $\lambda^{(2)}$ goes to negative to make the subtraction mean- ingful. The optimal $\lambda^{(2)}$ given by (33) depends on the particular channel parameters as well as the average BER of previous stage. However, (33) can be approximated by

$$
\begin{aligned}
\lambda_{\mathrm{opt}}^{(2)}= & \frac{\sum_{\substack{p=0 \\
p \neq r}}^{R-1} \Omega_{p}\left(L-2 \bar{B}^{(1)}\right)}{\sum_{\substack{p=0 \\
p \neq r}}^{R-1} \Omega_{p} L+\sum_{\substack{p=0 \\
p \neq r}}^{R-1}\left(\sigma_{p}^{(1)}\right)^{2}(L+g)} \\
\approx & \frac{\sum_{p=0}^{R-1} \Omega_{p}\left(L-2 L P_{e}^{(1)}\right)}{\sum_{p=0}^{R-1} \Omega_{p} L+\sum_{p=0}^{R-1}\left(\sigma_{p}^{(1)}\right)^{2}(L+g)}=\frac{1-2 P_{e}^{(1)}}{1+\delta^{(1)}}
\end{aligned}
$$

where

$$
\delta^{(1)}=(1+g / L) \sum_{p=0}^{R-1}\left(\sigma_{p}^{(1)}\right)^{2} / \sum_{p=0}^{R-1} \Omega_{p}
$$

In the case of perfect channel estimation, i.e., $\left(\sigma_{p}^{(1)}\right)^{2}=0$, or if the channel estimation error $\left(\sigma_{p}^{(1)}\right)^{2}$, given by (27), is much smaller than $\Omega_{p}, \delta^{(1)}$ is very small, and (34) can be approximated as

$$
\lambda_{\mathrm{opt}}^{(2)} \leq 1-2 P_{e}^{(1)}
$$

\section{Decision Statistics in More Stages}

The analytical results of the second stage can be extended to more stages without difficulty. The regenerated interference of the $r$ th finger in the $k$ th stage is given by

$$
\begin{aligned}
\tilde{v}_{r}^{(k)}(n)=\lambda^{(k)} \cdot \sum_{\substack{p=0 \\
p \neq r}}^{R-1} \frac{\tilde{\gamma}_{p}^{(k-1)}(m)}{2 \sqrt{g} N_{0}} \tilde{D}^{(k-1)}\left(n-n_{p}\right) \\
\quad \cdot \tilde{C}_{s}\left(n-n_{p}\right), \quad 0 \leq r \leq R-1
\end{aligned}
$$

where $\tilde{D}^{(k-1)}(n)=\sum_{l=1,3,5, \ldots} d_{l}^{(k-1)}(m) C_{l}(n)+$ $j \sum_{l=0,2,4, \ldots} d_{l}^{(k-1)}(m) C_{l}(n), 0 \leq l \leq L$.

The correlator output in the $l$ th data channel of the $k$ th stage is

$$
\begin{aligned}
\tilde{x}_{r, l}^{(k)}(m)= & \sum_{n=m N_{1}+1}^{(m+1) N_{1}}\left[\tilde{u}_{r}(n)-\tilde{v}_{r}^{(k)}(n)\right] \\
& \cdot \tilde{C}_{s}^{*}\left(n-n_{r}\right) \cdot(-j)^{\overline{l+1}} C_{l}\left(n-n_{r}\right) \\
= & \tilde{E}_{r, l}^{(1)}(m)+\tilde{I}_{r, l}^{(k)}(m)+\tilde{\eta}_{r, l}^{(1)}(m) .
\end{aligned}
$$

The variance of $\tilde{x}_{r, l}^{(k)}(m)$ is of the form

$$
\operatorname{var}\left\{\tilde{x}_{r, l}^{(k)}(m)\right\}=(1+j) N_{1} \Lambda^{(k)}
$$


where $\Lambda^{(k)}$ is given by

$$
\begin{aligned}
\Lambda^{(k)}=\frac{\Delta E}{2}( & \sum_{p=R}^{P-1} \Omega_{p}(L+g) \\
& +\sum_{\substack{p=0 \\
p \neq r}}^{R-1} \Omega_{p}\left[4 \lambda^{(k)} \bar{B}^{(k-1)}+\left(1-\lambda^{(k)}\right)^{2} L\right] \\
& \left.+\sum_{\substack{p=0 \\
p \neq r}}^{R-1}\left(\lambda^{(k)}\right)^{2}\left(\sigma_{p}^{(k-1)}\right)^{2}(L+g)\right)+\eta_{0} \\
\approx \frac{\Delta E}{2}\left(\sum_{p=R}^{P-1} \Omega_{p}(L+g)\right. & +\sum_{p=0}^{R-1} \Omega_{p}\left[4 \lambda^{(k)} \bar{B}^{(k-1)}+\left(1-\lambda^{(k)}\right)^{2} L\right] \\
& \left.+\sum_{p=0}^{R-1}\left(\lambda^{(k)}\right)^{2}\left(\sigma_{p}^{(k-1)}\right)^{2}(L+g)\right)+\eta_{0}
\end{aligned}
$$

and

$\left(\sigma_{p}^{(k-1)}\right)^{2}=\frac{2 \Lambda^{(k-1)}}{\Delta E g N_{P} N_{0}}, \quad k>2$.

The optimal cancellation factor of the $k$ th stage, $\lambda_{\mathrm{opt}}^{(k)}$, is of the form

$$
\lambda_{\mathrm{opt}}^{(k)}=\frac{1-2 P_{e}^{(k-1)}}{1+\delta^{(k-1)}} \leq 1-2 P_{e}^{(k-1)}
$$

where

$$
\delta^{(k-1)}=(1+g / L) \sum_{p=0}^{R-1}\left(\sigma_{p}^{(k-1)}\right)^{2} / \sum_{p=0}^{R-1} \Omega_{p} .
$$

\section{System Performance by BER}

When hard decision is applied and no channel coding is used, the instantaneous bit error probability of $l$ th branch data in the $k$ th stage is in similar form as that of the first stage, with substitution of $\Lambda^{(1)}$ by $\Lambda^{(k)}$. That is

$$
\begin{aligned}
P_{e}^{(k)} & =P_{e, l}^{(k)}\left(\zeta_{R}\right)=Q\left(\frac{\left|E\left\{z_{l}^{(k)}(m)\right\}\right|}{\sqrt{\operatorname{var}\left\{z_{l}^{(k)}(m)\right\}}}\right) \\
& =Q\left(\frac{\zeta_{R}}{\sqrt{\zeta_{R}\left(1+\frac{1}{g N^{\prime}}\right) \frac{\Lambda^{(k)}}{N_{1} E}+\frac{2 R}{g N^{\prime}}\left(\frac{\Lambda^{(k)}}{N_{1} E}\right)^{2}}}\right)
\end{aligned}
$$

where $\Lambda^{(k)}$ is given by (40). The error probability is conditioned on instantaneous multipath fading parameter $\zeta_{R}$. In order to get

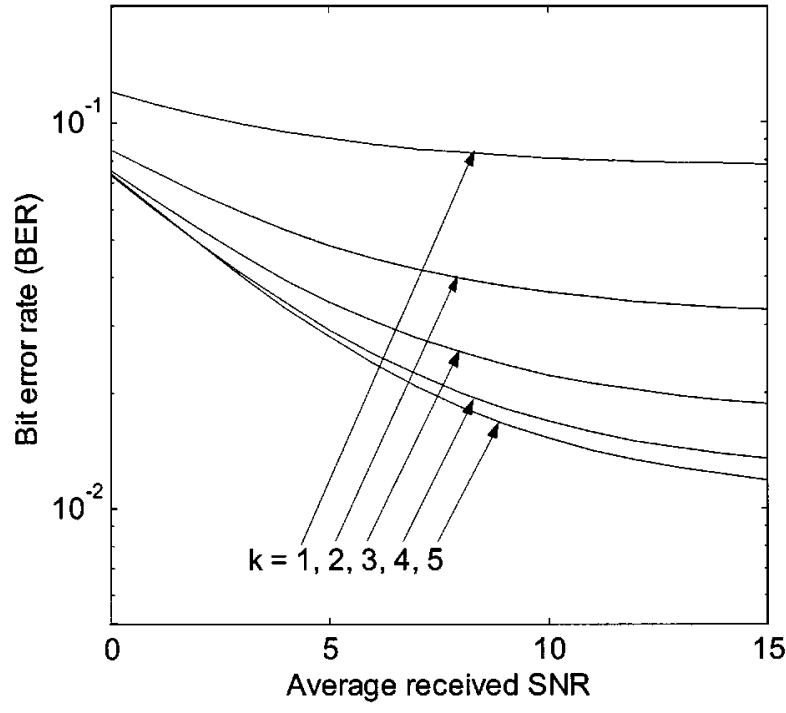

Fig. 6. BER versus SNR with increase of number of stages in system.

an average BER of the final decisions, the conditioned error probability should be averaged over the distributions of $\zeta_{R}$. The final average BER of the proposed system is given by

$$
P_{e}=\int_{0}^{\infty} P_{e}^{(k)}\left(\zeta_{R}\right) \cdot p\left(\zeta_{R}\right) d \zeta_{R}
$$

where $P_{e}^{(k)}$ is given by (48), and $p\left(\zeta_{R}\right)$ is of the form

$$
p\left(\zeta_{R}\right)=\left(\frac{m_{R}}{\Omega_{R}}\right)^{m_{R}} \frac{\zeta_{R}^{m_{R}-1}}{\Gamma\left(m_{R}\right)} \exp \left(-\frac{m_{R}}{\Omega_{R}} \zeta_{R}\right)
$$

where $\Omega_{R}=\sum_{r=0}^{R-1} \Omega_{r}=\sum_{r=0}^{R-1} E\left\{\alpha_{r}^{2}\right\}$ and $m_{R}=$ $\sum_{r=0}^{R-1} m_{r}$

\section{NUMERICAL RESULTS}

In this section, the effects of different system parameters on the BER performance of the multicode W-CDMA system are investigated by numerical calculations. Unless noted otherwise, the exponential decay rate of the MIP $\delta=0.2$ and the ratio $m_{l} / \Omega_{l}=4$ for all paths. The number of multipaths $P=8$, and the number of RAKE fingers $R=4$. The SF for pilot channel $N_{0}=256$, and for data channel $N_{1}=4$. The number of data channels for the reference user $L=6$. The number of LPF taps $N_{P}=2$ and the power ratio of pilot channel $g=0.3$. The SNR is defined as $\mathrm{SNR}=N E / \eta_{0}$, which is actually the average received SNR in the first path.

In Fig. 6, the BER performance is plotted versus the received SNR for different number of stages. The first stage $(k=1)$ is the BER without MCI cancellation. It is seen that the MCI cancellation effectively reduces the BER. The improvement is quite significant at stage two and stage three, but little improvement is observed beyond stage four. Therefore, in practical system, a receiver with three or four stages would be sufficient. Also, note that the cancellation factor in each stage is roughly chosen in this plotting according to the error rate. The values are 0 , $0.5,0.7,0.85$, and 0.95 for $k=1,2,3,4$, and 5 , respectively. They should be considered as typical values rather than optimal 


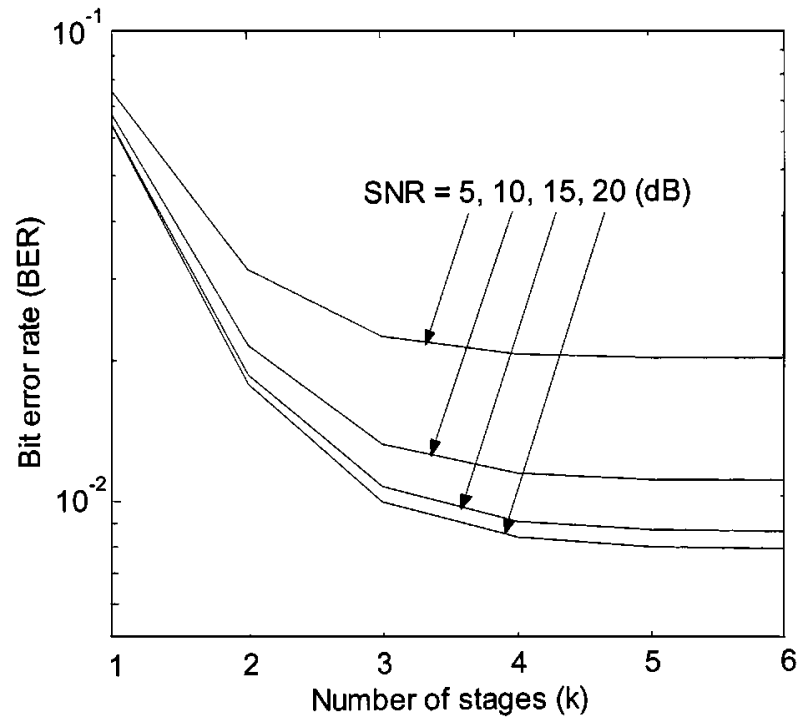

Fig. 7. BER versus number of stages with different SNR level.

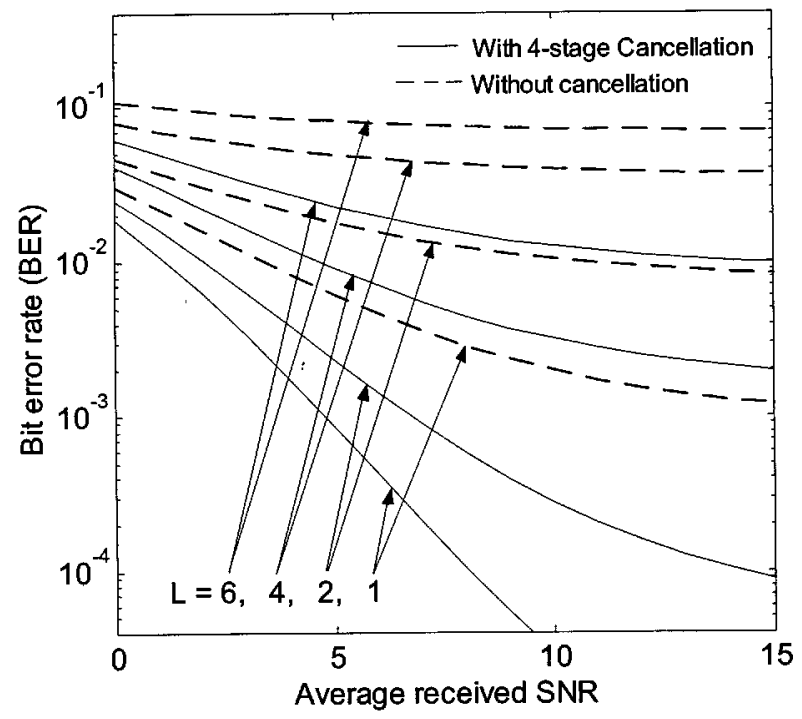

Fig. 8. BER versus SNR with different number of data channels.

values. As it is shown in the following figures, these values are slightly shifted from the optimal ones. The BER is also shown in Fig. 7 as a function of the number of stages. It can be seen that four stages are necessary for IC. This is consistent with that obtained from Fig. 6.

Fig. 8 shows the system BER performance in terms of received SNR with different number of data channels. The system utilizes a four-stage $\mathrm{MCI}$ cancellation receiver with cancellation factor set to be $0,0.5,0.75$, and 0.95 , respectively. The performance degradation due to the increasing number of data channels is obvious. However, acceptable performance (BER $\approx$ $10^{-2}$ ) can be achieved even if total six data channels are in use.

In Fig. 9, the BER is plotted versus SNR with different number of RAKE fingers $R$. The receiver is also of four stages with cancellation factor set as $0,0.5,0.75$, and 0.95 , respectively. It is clearly shown that the more RAKE finger is utilized, the more advantage we can get from MCI cancellation. This is because when more RAKE fingers are used, more multipaths

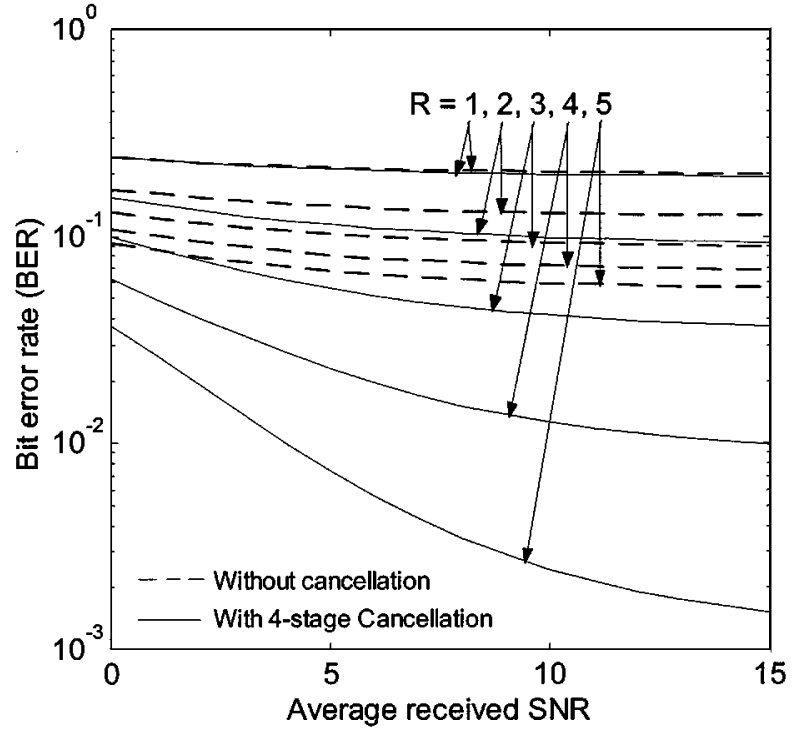

Fig. 9. BER versus SNR with different number of RAKE fingers.

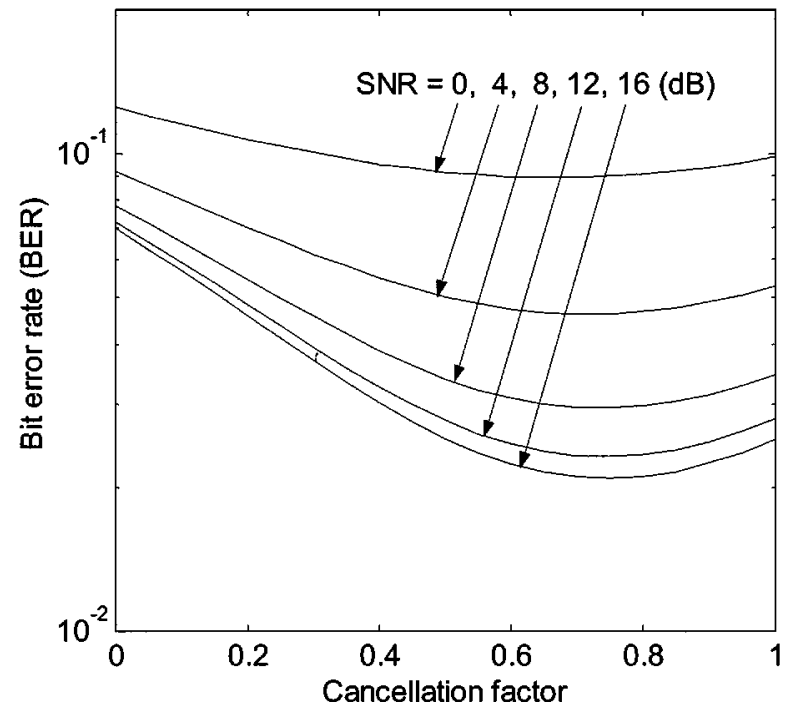

Fig. 10. BER versus cancellation factor with different SNR level.

are estimated and the MCI in these multipaths can be cancelled and energy in more paths can be employed for data detection.

In Fig. 10, the BER performance is plotted versus the cancellation factor. The curves are differed from each other in SNR level. In order to obtain simple conclusion, only a two-stage MCI cancellation receiver is used when plotting this figure. There is no cancellation in the first stage as it should be, and the cancellation factor at the second stage is varied from zero to one to show the distinct results. As expected, the increase of cancellation factor gradually decrease the BER until it reaches an optimal value, then the BER increases again. The situations are the same for different SNR level, but the optimal value may slightly shift from each other.

Fig. 11 illustrates the optimal cancellation factor $\lambda_{\text {opt }}^{(k)}$, given by (42), as the function of received SNR level for different stages (i.e., $k=2,3,4$, and 5). As it is seen from the figure, when the SNR is low, the optimal cancellation factor is small, while a higher SNR requires a higher cancellation factor. It is also 


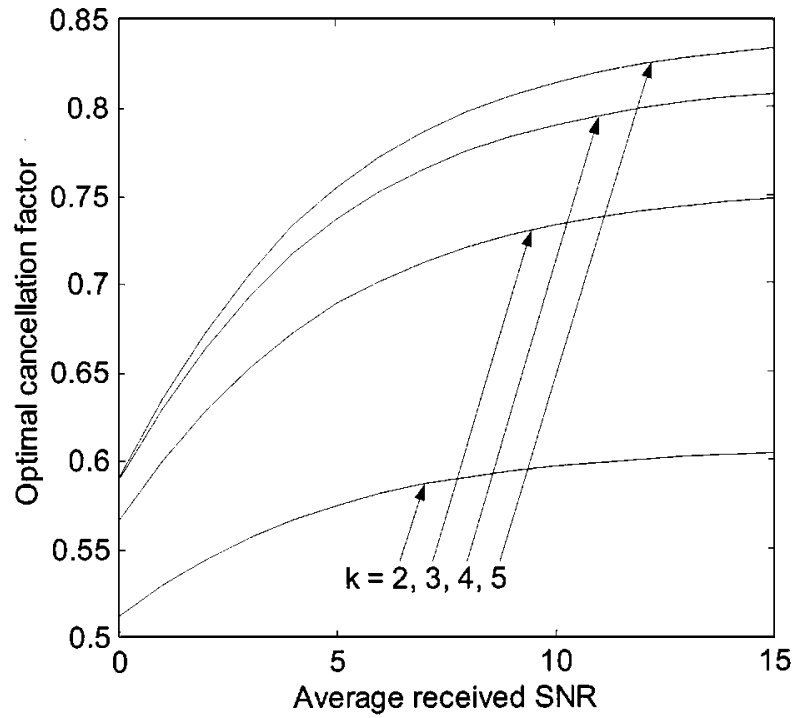

Fig. 11. Optimal cancellation factor versus SNR in different stage.

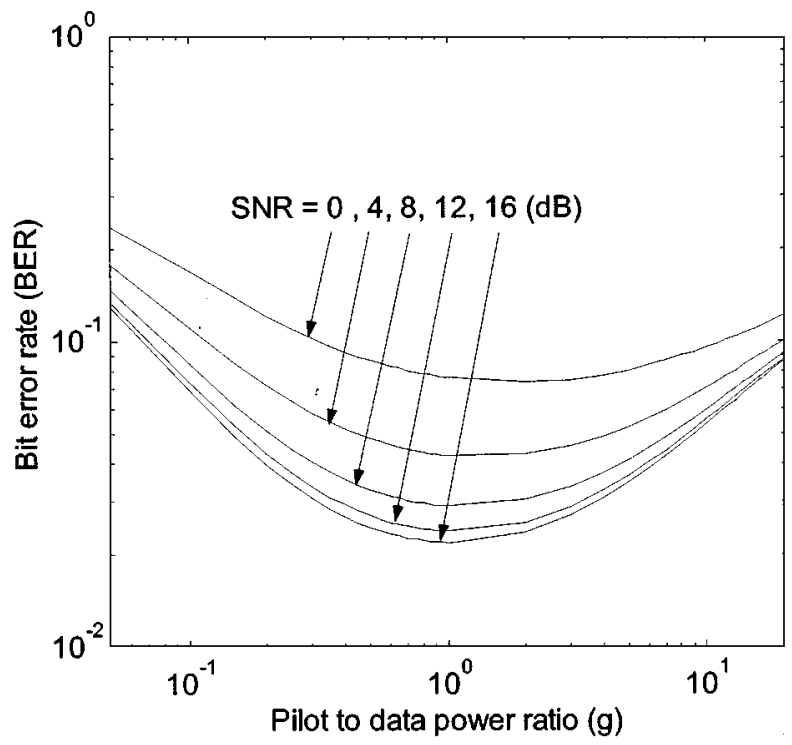

Fig. 12. BER versus pilot to data power ratio with different SNR level.

shown that with the increase of number of stages, the optimal cancellation factor goes larger. The reason is that the BER is reduced with the increase of stages. However, same as what we found in Figs. 6 and 7, there is little performance improvement between stages four and five, which result in little changes of the optimal cancellation factor. The optimal value of the cancellation factor should be chosen from 0.5 to 0.85 for a wide range of signal to noise ratio, as observed from the figure.

In Fig. 12, the relationship between BER performance and pilot to data power ratio $g$ is examined. It is seen from the figure that too small or too large values of $g$ lead to worse performance. An optimal value of pilot to data power ratio exists according to different SNR level. This optimal value is found to be near 1.2 under the given parameters. That is, the optimal power ratio of pilot to total data channels is $1.2 / 6 \approx-7 \mathrm{~dB}$, which is in consistence with that in [12].

\section{CONCLUSION}

This paper presents a multicode RAKE receiver with MCI cancellation for high and flexible rate data transmission over multipath fading channels. The following conclusions are drawn:

1) MCI cancellation can effectively reduce the BER in multipath environment. With a four-stage receiver, the BER can be lowered by approximately an order $(\mathrm{BER} \approx$ $10^{-1} \rightarrow \mathrm{BER} \approx 10^{-2}$ ).

2) The optimal cancellation factor of each stage is gradually increased with the decreasing of BER. The optimal cancellation factor in the $k$ th stage is approximated by $\lambda_{\text {opt }}^{(k)} \leq 1-2 P_{e}^{(k-1)}$, where $P_{e}^{(k-1)}$ is the error probability of the $(k-1)$ th stage. The values of these factors can be roughly chosen from 0.5 to 0.85 for different signal to noise ratios.

\section{ACKNOWLEDGMENT}

The authors would like to thank K. Higuchi of NTT DoCoMo, Japan, and B. Xia of University of Hong Kong for their helpful discussions.

\section{REFERENCES}

[1] I Chih-Lin and R. D. Gitlin, "A profile-based location strategy and its performance," IEEE J. Select. Areas Commun., vol. 15, pp. 1415-1424, Oct. 1997.

[2] F. Adachi, K. Ohno, A. Higashi, T. Dohi, and Y. Okumura, "Coherent multicode DS-CDMA mobile radio access," IEICE Trans. Commun., vol. E79-B, pp. 1316-1325, Sept. 1996.

[3] Spreading and Modulation (FDD). 3GPP TSG-RAN, 2000-06.

[4] N. Guo and L. B. Milstein, "On rate-variable multidimensional DS/SSMA with sequence sharing," IEEE J. Select. Areas Commun., vol. 17, pp. 902-917, May 1999.

[5] Z. Liu, M. J. Karol, M. E. Zarki, and K. Y. Eng, "Interference issues in multicode CDMA networks," in Proc. IEEE PIMRC'96, vol. 1, Oct. 1996, pp. 98-102.

[6] H. D. Schotten, H. Elders-Boll, and A. Busboom, "Adaptive multicode CDMA systems for variable data rates," in Proc. IEEE ICPWC'97, Dec. 1997, pp. 334-337.

[7] D. N. Kalofonos, E. Kurtas, and J. G. Proakis, "Performance of multicode CDMA systems with concatenated coding and iterative decoding in multipath Rayleigh fading channels," in Proc. IEEE VTC'99-Fall, vol. 5, Sept. 1999, pp. 2566-2570.

[8] D. Ayyagari and A. Ephremides, "Cellular multicode CDMA capacity for integrated (voice and data) services," IEEE J. Select. Areas Commun., vol. 17, pp. 928-938, May 1999.

[9] T. Ottosson, "Precoding in multicode DS-CDMA systems," in Proc. IEEE ISIT'97, June 1997, p. 351.

[10] N. Guo and L. B. Milstein, "Uplink performance evaluation of multicode DS/CDMA systems in the presence of nonlinear distortions," IEEE J. Select. Areas Commun., vol. 18, pp. 1418-1428, Aug. 2000.

[11] D. I. Kim and K. H. Park, "A hybrid nonlinear distortion compensator for multicode DS/CDMA systems," in Proc. IEEE ICUPC'98, vol. 2, Oct. 1998, pp. 1087-1091.

[12] J. Wang and J. Chen, "Performance of wideband CDMA systems with complex spreading and imperfect channel estimation," IEEE J. Select. Areas Commun., vol. 19, pp. 152-163, Jan. 2001.

[13] A. C. K. Soong and W. A. Krzymien, "A novel CDMA multiuser interference cancellation receiver with reference symbol aided estimation of channel parameters," IEEE J. Select. Areas Commun., vol. 14, pp. 1536-1547, Oct. 1996

[14] M. Sawahashi, H. Andoh, and K. Higuchi, "Interference rejection weight control for pilot symbol-assisted coherent multistage interference canceller using recursive channel estimation in DS-CDMA mobile radio," IEICE Trans. Fundamentals, vol. E81-A, pp. 957-972, May 1998. 
[15] N. S. Correal, R. M. Buehrer, and B. D. Woerner, "Real-time DSP implementation of a coherent partial interference cancellation multiuser receiver for DS-CDMA," in Proc. IEEE ICC'98, vol. 3, June 1998, pp. $1536-1540$.

[16] P. K. Frenger, P. Orten, and T. Ottosson, "Code-spread CDMA with interference cancellation," IEEE J. Select. Areas Commun., vol. 17, pp. 2090-2095, Dec. 1999.

[17] M. C.-C. Chan and J. C.-I. Chuang, "Multicode high-speed transmission with interference cancellation for wireless personal communications," in Proc. IEEE VTC'96, vol. 2, May 1996, pp. 661-665.

[18] R. Herzog, "Interference cancellation for a high data rate user in coded CDMA systems," in Proc. IEEE ICC'98, vol. 2, June 1998, pp. 709-713.

[19] D. Koulakiotis and A. H. Aghvami, "Performance enhancement of multicode CDMA using interference cancellation," in Proc. IEEE ISSTA'98, vol. 1, Sept. 1998, pp. 130-134.

[20] Y. Suzuki and K. Kobayashi, "Interference cancellation method for DS-CDMA multicode-packet transmission," in Proc. IEEE GLOBECOM'98, vol. 6, Nov. 1998, pp. 3578-3583.

[21] S. I. Kim and H. S. Lee, "Performance improvement of RAKE receiver for a multicode DS-CDMA system with multistage interference cancellation detectors," in Proc. IEEE TENCON'99, vol. 1, Sept. 1999, pp. 573-576.

[22] S. Tanaka, H. Harada, M. Sawahashi, and F. Adachi, "Experiments on coherent adaptive antenna array diversity for wideband DS-CDMA mobile radio," IEEE J. Select. Areas Commun., vol. 18, pp. 1495-1504, Aug. 2000

[23] E. K. Al-Hussaini and A. A. M. al-Bassiouni, "Performance of MRC diversity systems for detection of signals with nakagami fading," IEEE Trans. Commun., vol. COMM-33, pp. 1315-1319, Dec. 1985.

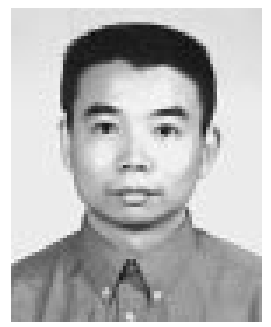

Jun Chen received the B. Eng. degree in electrical engineering and the M. Eng. degree in information and communication engineering from the University of Science and Technology of China, Hefei, China, in 1996 and 1999, respectively. He is currently pursuing the Ph.D. degree in electrical engineering at the University of Hong Kong, Hong Kong.

From 1995 to 1999 , he was with the Personal Communication and Spread Spectrum Laboratory, the University of Science and Technology of China, as a Research Engineer involved in the development of prototype CDMA communication systems. His research interests are in the area of mobile communication system, wireless networking, and VLSI implementation of wireless transceiver.

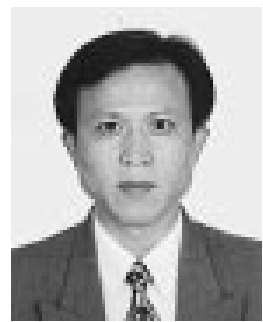

Jiangzhou Wang (M'91-SM'94) received the B.S. and M.S. degrees from Xidian University, Xian, China, in 1983 and 1985, respectively, and the Ph.D. degree (with Greatest Distinction) from the University of Ghent, Ghent, Belgium, in 1990, all in electrical engineering.

From 1990 to 1992, he was a Postdoctoral Fellow in the University of California, San Diego, where he worked on the research and development of cellular CDMA systems. From 1992 to 1995 , he was a Senior System Engineer at Rockwell International Corporation, Newport Beach, CA, where he worked on the development and system design of wireless communications. Since 1995, he has been with the University of Hong Kong, Hong Kong, where he is currently an Associate Professor. He has held a Visiting Professor position in NTT DoCoMo, Japan. He has published over 100 papers, including more than 20 IEEE Transactions/Journal papers in the areas of wireless mobile and spread spectrum communications. He has written/edited two books, entitled "Broadband Wireless Communications" (Kluwer, 2001) and "3G Mobile Enhanced Technologies" (Artech House, 2001), respectively. He holds one US patent in the GSM system.

Dr. Wang was a Technical Chairman of IEEE Workshop in 3G Mobile Communications, 2000. He is an Editor for IEEE TRANSACTIONS ON COMMUNICATIONS and a Guest Editor for IEEE JOURNAL ON SELECTED AREAS In COMMUNICATIONS. He is listed in Who's Who in the World.

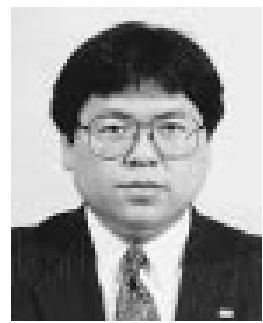

Mamoru Sawahashi (M'88) received the B.S. and M.S. degrees from Tokyo University, Tokyo, Japan, in 1983 and 1985, respectively, and received the Dr. Eng. Degree from the Nara Institute of Technology, Japan, in 1998.

In 1985 he joined NTT Electrical Communications Laboratories, and in 1992 he transferred to NTT Mobile Communications Network, Inc. (now, NTT DoCoMo, Inc.). Since joining NTT, he has been engaged in the research of modulation/demodulation techniques for mobile radio, and research and development of wireless access technologies for W-CDMA mobile radio and broadband wireless packet access technologies for beyond IMT-2000. He is now the Director of the Wireless Access Laboratory of NTT DoCoMo, Inc. 